\title{
Random Message Arrivals in a Gaussian Cognitive Radio
}

\author{
Sriram N. Kizhakkemadam and Dinesh Rajan \\ Dept. of Electrical Engineering \\ Southern Methodist University \\ Dallas TX 75206 \\ Email: \{skizhakk, rajand\}@engr.smu.edu
}

\begin{abstract}
We study the impact of random message arrivals in a two user Gaussian interference channel with one-way cooperation, also called as cognitive radio. In this model, one user (labeled cognitive user) has information about the message of the other user (labeled primary user). Specifically, we quantify the reduction in queuing delay for both the primary and cognitive users when the cognitive user has either non-causal or causal information about primary users data. Surprisingly, we find that the delay for the primary user also reduces even though the cognitive user does not use any of its transmission power to relay the information of the primary user.
\end{abstract}

\section{INTRODUCTION}

Cognitive radio encompasses a broad range of technologies in which one user(s) labeled the cognitive user(s) shares the spectrum resource of another user labeled as the primary user [7], [8]. The capacity region of cognitive radio for a Gaussian channel has been studied [3, 4]. In both [3] and [4], the primary and cognitive users are assumed to be always transmitting information. A key reason for consideration of cognitive radio is the presence of spectrum holes [7] due to periods of inactive transmission by the primary user. These spectrum holes could arise due to the random arrival of messages at user terminals. It is therefore pertinent to study the impact of random message arrivals in a cognitive radio.

In [3], the cognitive radio channel is modeled as an interference channel with one-way co-operation from the primary transmitter to the cognitive transmitter. We add random message arrival to this model. More specifically, in our model of cognitive radio, a primary user, $X_{p}$ transmits its message to an intended receiver, $Y_{p}$. The cognitive user, $X_{c}$ likewise transmits its message to its intended receiver, $Y_{c}$. These two simultaneous transmissions interfere with each other as shown in Fig. 1(a). A high-capacity one-way link is assumed to exist from the primary to the cognitive user (possibly due to proximity). While the cognitive user has access to the primary user's information (either causally or non-causally), the reverse link is not possible. The cognitive user can make use of the side-information of the primary user to perform an interference cancellation or alter its transmission strategy to mitigate interference at its receiver. Thus, side-information is useful for the cognitive user. We first show a dirty paper like coding scheme [1] that allows the cognitive user to achieve rates as if the primary user is absent even when messages arrive randomly. In [3] the cognitive user is allowed to transmit the information of the primary user and thereby increase the achievable rates for the primary user. In contrast, we show that even without the cognitive user transmitting the primary user's information, the transmission rates of the primary user increases with random message arrivals. Intuitively, if the cognitive user is allowed to service its messages sooner, the primary user sees lesser interference on an average. This leads to an increase in the average rate for the primary user.

\section{SySTEM MOdeL}

Consider messages arriving at random at both the primary and cognitive transmitter according to a Poisson arrival process with average arrival rate of $\lambda$ messages per second. These messages are stored in a queue at the transmitter. In order to avoid a cumbersome analysis of message queues at the transmitter, following [10], we treat every new message as a new virtual user. The buffer capacity at the transmitter is assumed to be infinite. This infinite user population assumption converts our channel from a point to point link between the primary user and its corresponding receiver to a Multiple Access Channel (MAC) with each virtual user of the MAC transmitting one message. Further, every virtual user sees the same channel. Therefore, the two user cognitive radio can be modeled as a twin MAC with the signal of one MAC interfering with the other as illustrated in Fig.1. Consequently, a linear system model for the cognitive radio can be written as,

$$
\begin{aligned}
& y_{p}=\sum_{i=1}^{u(t)} \sqrt{\mathrm{SNR}_{p}} x_{p}(i)+\sum_{j=1}^{v(t)} \sqrt{\gamma_{c} \mathrm{SNR}_{c}} x_{c}(j)+z_{p} \\
& y_{c}=\sum_{k=1}^{v(t)} \sqrt{\operatorname{SNR}_{c}} x_{c}(k)+\sum_{l=1}^{u(t)} \sqrt{\gamma_{p} \operatorname{SNR}_{p}} x_{p}(l)+z_{c}
\end{aligned}
$$

The subscripts $p$ and $c$ denote the primary and cognitive user respectively. The number of messages in the queue of the primary and the cognitive user at time $t$ is denoted by $u(t)$ and $v(t)$ respectively. The time index is dropped when it is readily understood from the context. The variables $x$ and $y$ represent a symbol of the transmitted and received codeword respectively (of either the primary or cognitive user with the appropriate subscript). All the transmitters and receivers are assumed to know the cross-over gains $\gamma_{p}$ and $\gamma_{c}$. The normalized input signals $x_{p}$ and $x_{c}$ and the additive noise at 


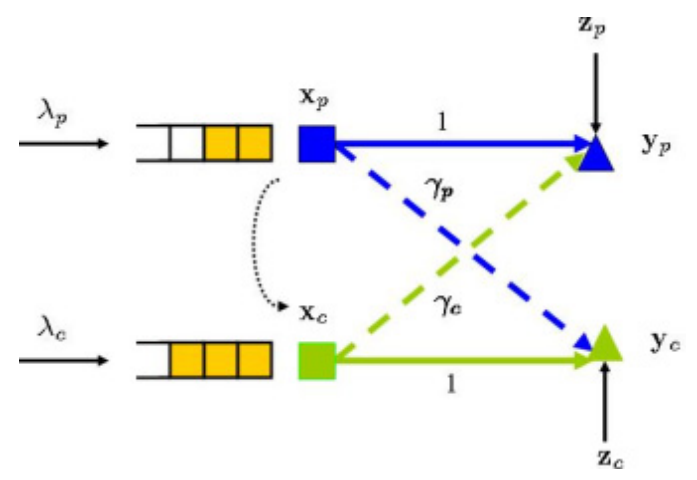

(a)

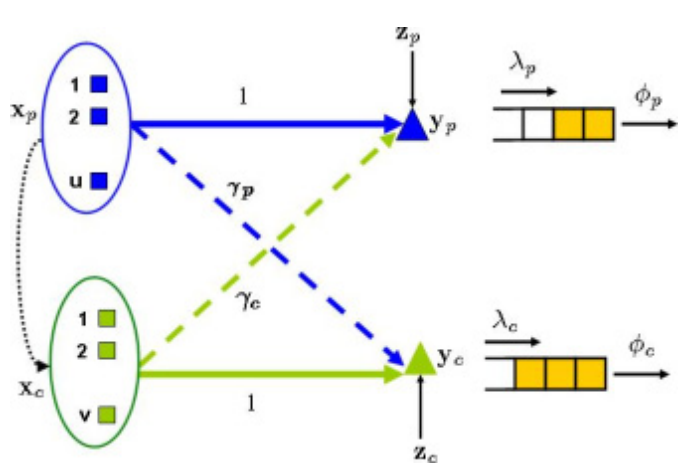

(b)

Fig. 1. (a) Interference model for a cognitive radio with random message arrival and one-way side-information, (b) Equivalent Multiple Access Channel

each of the receivers, $z_{p}$ and $z_{c}$ are drawn from a complex Gaussian distribution according to $\mathcal{C N}(0,1)$. The bandwidth is denoted by $W$. Each message for the primary user is chosen from an index set, $m_{p} \in\left\{1,2, \ldots, K_{p}\right\}$. Likewise, the cognitive user chooses a message from $m_{c} \in\left\{1,2, \ldots, K_{c}\right\}$ for transmission. We assume a uniform distribution for the messages over their respective support sets. The cognitive user has access to both its message and that of the primary user, either by a non-causal or a causal scheme. The total number of messages in the queue is assumed to be finite leading to finite power constraints for both primary and cognitive users if the stability conditions are satisfied (Section IV). The primary and the cognitive users reveal the number of messages in their queue to the respective receiver, possibly by signaling over an orthogonal low rate channel.

\section{COMBINED QUEUING THEORY AND INFORMATION THEORY}

In this section, we delineate the analysis introduced by Telatar and Gallager in [10] to model random message arrivals in a multiple access channel. This analysis is valid for both the primary and cognitive user and so we drop the subscripts. If $m(t)$ messages are in the transmitter's queue, each message is encoded into a possibly infinite length codeword and transmitted. A processor sharing model is assumed that allocates equal service for all the messages in the queue. Successive symbols of the codeword are sent until the transmitter receives a feedback from the receiver that the message is decoded. Thus, only a finite length of codeword is required for each transmitter. In the limit of large block lengths to achieve a small probability of error, this nominal feedback is neglected in the analysis. Due to the equivalent virtual user model, the queue is analyzed as comprising of $m(t)$ active messages waiting for service at the primary receiver [10]. For this equivalent queue at the receiver, the arrival rate for each of the messages is still $\lambda$. However the service rate is the sum service rate of all the messages (since the equivalent queue at the receiver has the messages of all the virtual users). The sum service rate for $m$ messages in the equivalent MAC is denoted as $\phi(m)$. Due to the processor sharing assumption, each message gets $\phi(m) / m$ amount of service. A standard M/M/1 model for the queue with Poisson arrivals and exponential service times is assumed. The equilibrium distribution of the number of messages in the $\mathrm{M} / \mathrm{M} / 1$ queue is given as [10],

$$
\begin{gathered}
p(m)=\frac{l^{m}}{C \phi_{!}(m)} \\
C=1+\sum_{i=1}^{\infty} \frac{l^{i}}{\phi_{!}(i)}, \quad \phi_{!}(m)=\Pi_{i=1}^{m} \phi(i)
\end{gathered}
$$

Recognize that $1 / C$ is the probability that there are no messages to be transmitted in the queue. Also, $\phi_{!}(0)=1$. The parameter $l$ is also called as the loading factor of the queue. The loading factor and the service rate are respectively related to the demand and supply of a queue. In [10], the theory of error exponents is used to relate the loading factor to the message size (demand) and the random coding error exponent term to the service rate (supply). Accordingly, the loading factor is given by [10] as $l \triangleq \lambda\left(m \log K-\log P_{e}\right) / W$.

For a Gaussian Multiple Access channel with joint decoding of $m(t)$ messages and a total interference power $P_{i}$, the random coding error exponent is given by [9],

$$
E_{0}=\rho \log \left(1+\frac{m S N R}{(1+\rho)\left(1+P_{i}\right)}\right)
$$

The service rate for the queue is given by [10] as,

$$
\phi(m)=W E_{o}(m, \mathrm{SNR})
$$

Note that the error exponent expressions are very similar to the sum rate capacity expressions for a Gaussian MAC. For a Gaussian MAC with equal power constraints for all users, if $R_{i}$ is the rate for the $i^{t h}$ user, the sum rate capacity with joint decoding is given by,

$$
R(m, \mathrm{SNR})=\sum_{i=1}^{m} R_{i} \leq \log (1+m \mathrm{SNR})
$$

If $\mathrm{SNR}^{\prime}=\mathrm{SNR} /(1+\rho)$, the sum rate capacity in $(7)$ and the service rate in (6) are related by,

$$
\phi(m)=W R\left(m, \mathrm{SNR}^{\prime} /\left(1+P_{i}\right)\right)
$$


The receiver computes the error exponent for each successive symbol of the codeword of every message and accumulates it. If the accumulated error exponent is greater than the demand $S=\log m-\log P_{e}$, it has sufficient number of symbols to decode the first codeword transmitted according to a ML decoding procedure. The above procedure is repeated for successive messages. Given the probability distribution of the number of messages in a MAC in (3), the queuing delay can be found using Little's law as,

$$
\bar{D}=\frac{\mathcal{E}[m]}{\lambda}=\frac{\sum_{m=1}^{\infty} m p(m)}{\lambda}=\frac{1}{\lambda} \sum_{m=1}^{\infty} \frac{m l^{m}}{C \phi_{!}(m)}
$$

\section{Non-Causal Gaussian Cognitive Radio}

The primary user shares its codeword with the cognitive user non-causally. With random message arrival, the interference from one of the users at the other user's receiver is time varying. The state of the queue (message index of each packet in the buffer) gives complete information of the messages being serviced. Instead of encoding each message separately, we equivalently encode the state of the queue. For the primary user, each buffer stores either one of the $K_{p}$ message indices or is empty. A buffer is empty if either no new message has arrived and if the messages in the buffer are already serviced. Therefore, for a $u^{*}$ length queue, the number of possible states of the queue would be $K_{p}^{u^{*}}+1$ (counting no message in the queue also as a state). The queue of the cognitive user likewise has $K_{c}^{v^{*}}+1$ possible states. The following lemma shows the gains for the cognitive radio in this setup.

Lemma 4.1: In a non-causal Gaussian cognitive radio with random message arrivals, the achievable rate for the cognitive user with $v$ messages in its queue and non-causal information of the state of the queue of the primary user is

$$
R_{c}\left(v, \mathrm{SNR}_{c}\right)=\lim _{n \longrightarrow \infty} \frac{v \log K_{c}}{n} \leq \log \left(1+v \mathrm{SNR}_{c}\right)
$$

Proof: Encoding: A unique codeword is generated for each state of the queue(s) for both users as ${ }^{1}$ :

$$
\begin{aligned}
E_{p}: & \left\{1,2, \ldots, K_{p}^{u}\right\} \longrightarrow \mathcal{X}_{p} \\
E_{c}: & \left\{1,2, \ldots, K_{p}^{u}+1\right\} \times\left\{1,2, \ldots, K_{c}^{v}\right\} \longrightarrow \mathcal{X}_{c}
\end{aligned}
$$

where, we have denoted the input alphabet to the channel of the primary and cognitive user as $\mathcal{X}_{p}$ and $\mathcal{X}_{c}$ respectively. Note that the codeword of the cognitive user depends on the state of the queue of both users which is not so for the primary user. Each element of the codeword is drawn i.i.d according to $\mathcal{C N}(0,1)$. This codeword is revealed to the respective receiver. Similar to [10], the length of the codeword is not fixed. It depends on the interference from the cognitive user and the desired upper bound on the probability of error. The transmitter keeps sending successive symbols of the codeword corresponding to the state of the queue(s). Depending on the number of the messages, the power level of the codeword is amplified to a power level of $u(t) \mathrm{SNR}_{p}$ for the primary

\footnotetext{
${ }^{1}$ Unlike our approach, each message is encoded separately in [10]
}

user and $v(t) \mathrm{SNR}_{c}$ for the cognitive user before transmission. The transmitted codeword is changed if either a new message arrives at its terminal or if the encoder receives a message from the primary decoder that the earliest message in the queue was decoded within the specified probability of error. Additionally, the cognitive user's transmitted codeword is changed if there is a change in the state of the queue of the primary user.

Decoding for Cognitive User: Based on the received signal strength and the information of the number of messages in the queue of its transmitter, the cognitive user computes and accumulates $E_{0}=W \log \left(1+v \mathrm{SNR}_{c}^{\prime}\right)$. If the error exponent is greater than the demand $v \log K_{c}-\log P_{e}$, the decoder decides that the number of symbols it has accumulated is sufficient for decoding (say, $n$ ). This $n$ length super-codeword is a concatenation of the codewords corresponding to various states in the interval. The decoder then decides the index of the transmitted codeword according to a maximum likelihood decoding (ML) procedure and a decoding function, $d: \mathcal{Y}_{c}^{n} \longrightarrow\left\{1,2, \ldots, K_{c}^{v}\right\}$. Unlike a regular ML decoder, the number of likelihood function computations keeps changing as the interference or number of messages in the transmitter's queue changes. It can be shown that by the random coding bound, the probability of error will be less than $P_{e}$ (proof omitted due to lack of space, [5]). After accumulating the symbols according to the error exponent criterion, the receiver then indicates to the transmitter that a message index was decoded. The cognitive user will then eliminate the earliest message index in the queue. In the limit of large block lengths, since all the $v$ messages of the cognitive user can be decoded, with joint decoding the cognitive user achieves the rate given in the lemma for any arbitrarily chosen $P_{e}$. This procedure is repeated to decode the remaining messages in the queue.

The decoding is similar for the primary user, except that due to the lack of information of the cognitive user's queue, it cannot mitigate the interference. Consequently, the average rate for the primary user satisfies [2]:

$$
\begin{aligned}
\sum_{i=1}^{u} R_{p}(i) & \leq \mathcal{E}_{v} I\left(X_{p 1}, \ldots, X_{p u} ; Y_{p}\right) \\
& =\mathcal{E}_{v} \log \left(1+\frac{u \mathrm{SNR}_{p}}{1+v \mathrm{SNR}_{c}}\right)
\end{aligned}
$$

where the expectation is over the distribution of the number of messages for the cognitive user. Due to the relation between sum service rate and sum rate capacity, we will make use of (14) and (10) to characterize the queuing behavior in Theorem 4.1 .

Theorem 4.1: In a non-causal Gaussian cognitive radio with random message arrivals, if the cognitive mitigates the primary user's interference, the average rate for the primary user increases.

Proof: From (8) and (10), the sum service rate for a cognitive user with non-causal information of the primary user's codeword and joint decoding of messages of the equivalent $\mathrm{MAC}$ is

$$
\phi_{c}(v)=W \log \left(1+v \mathrm{SNR}_{c}^{\prime}\right)
$$


If the genie information is not available for the cognitive user, the sum service rate with joint decoding of the individual messages is obtained from (8) and (14) as,

$$
\widetilde{\phi}_{c}(v \mid u)=W \log \left(1+\frac{v \mathrm{SNR}_{c}^{\prime}}{1+u \mathrm{SNR}_{p}}\right)
$$

The probability distribution of the number of messages for the cognitive user with genie information is obtained by indexing the generic expression in (3) to yield,

$$
p_{c}(v)=\frac{l_{c}^{v}}{C_{c} \phi_{v_{!}}(v)}, \quad C_{c}=1+\sum_{j=1}^{\infty} \frac{l_{c}^{j}}{\phi_{v_{!}}(j)}, \quad v \in[0, \infty]
$$

Likewise, the probability distribution for the cognitive user without genie information is,

$\widetilde{p}_{c}(v \mid u)=\frac{\widetilde{l}_{c}^{v}}{\widetilde{C}_{c}(u) \widetilde{\phi}_{v_{!}}(v \mid u)}, \widetilde{C}_{c}(u)=1+\sum_{j=1}^{\infty} \frac{\widetilde{l}^{j}}{\widetilde{\phi}_{v_{!}}(j \mid u)}, v \in[0$,

For the primary user, if the cognitive user has genie information, the sum service rate is given by,

$$
\phi_{p}(u)=\mathcal{E}_{p_{c}} \Psi(u, v)
$$

If the genie information is not available for the cognitive user, the sum service rate is,

$$
\widetilde{\phi}_{p}(u)=\mathcal{E}_{\widetilde{p}_{c}} \Psi(u, v)
$$

where

$$
\Psi(u, v)=W \log \left(1+\frac{u \mathrm{SNR}_{p}^{\prime}}{1+v \mathrm{SNR}_{c}}\right)
$$

The notations for the loading factor are distinct for analysis of stability condition for the queue. From (15) and (16), we know that if $u \geq 1, \phi_{c}(v)>\widetilde{\phi}_{c}(v \mid u)$ for all positive $\mathrm{SNR}_{c}, \mathrm{SNR}_{p}$. Due to the inverse relationship between the normalizing constant and the sum rate capacity, $\widetilde{C}_{c}(u)>C$ We use this relationship between the normalizing constant to prove in the footnote that $\phi_{p}(u)>\widetilde{\phi}_{p}(u) \forall u$. Thus, the average sum service rate for the primary user is greater if it shares its information with the cognitive user than otherwise.

Since the average service rate improves with sharing of the codeword, the sum rate capacity which is just a scaled version of the sum service rate also increases with genie information for the cognitive user. We can easily show that an increase in sum service rate translates to decrease in queuing delay [5]. Representative numerical plots are given in Section V

Stability Criteria: By Loynes criterion [6], the queue of the system is said to be stable if the arrival rate is less than the service rate. A sufficient condition for the stability of the primary and the cognitive user's queue is, $l_{p}<\log \left(1+\mathrm{SNR}_{p}\right)$ and $l_{c}<\log \left(1+\mathrm{SNR}_{c}\right)$. Detailed analysis can be found in [5]. If the cognitive user were not to have non-causal information of the primary user's codeword, $\widetilde{l}<1$ for stability

$$
\begin{aligned}
& \text { Consider }\left(\frac{1}{C}-\frac{1}{\widetilde{C}_{c}(u)}\right) \log \left(1+u \mathrm{SNR}_{p}^{\prime}\right)=\left[\frac{1}{1+\sum_{j=1}^{\infty} \frac{l^{j}}{\phi_{v_{!}}(j)}}-\frac{1}{1+\sum_{j=1}^{\infty} \frac{\widetilde{l}^{j}}{\bar{\phi}_{v_{!}}(j \mid u)}}\right] \log \left(1+u \mathrm{SNR}_{p}\right) \\
& =\frac{1}{C \widetilde{C}_{c}(u)}\left(\sum_{j=1}^{\infty} \frac{\widetilde{l}^{j}}{\widetilde{\phi}_{v_{!}}(j \mid u)}-\frac{l^{j}}{\phi_{v_{!}}(j)}\right) \log \left(1+u \mathrm{SNR}_{p}\right) \\
& >\frac{1}{C \widetilde{C}_{c}(u)} \sum_{j=1}^{\infty}\left(\frac{\widetilde{l}^{j}}{\widetilde{\phi}_{v !}(j \mid u)}-\frac{l^{j}}{\phi_{v !}(j)}\right) \log \left(1+\frac{u \mathrm{SNR}_{p}}{1+j \mathrm{SNR}_{c}}\right) \\
& \Rightarrow\left(\frac{1}{C}-\frac{1}{\widetilde{C}_{c}(u)}\right) \log \left(1+u \mathrm{SNR}_{p}\right)+\frac{1}{C \widetilde{C}_{c}} \sum_{j=1}^{\infty}\left(\frac{l^{j}}{\phi_{v_{!}}(j)}-\frac{\widetilde{l}^{j}}{\widetilde{\phi}_{v !}(j \mid u)}\right) \log \left(1+\frac{u \mathrm{SNR}_{p}}{1+j \mathrm{SNR}_{c}}\right)>0 \\
& \Rightarrow\left(\frac{1}{C}-\frac{1}{\widetilde{C}_{c}(u)}\right) \log \left(1+u \mathrm{SNR}_{p}\right)+\frac{1}{C \widetilde{C}_{c}(u)} \sum_{j=1}^{\infty}\left(\frac{\widetilde{C}_{c}(u) l^{j}}{\phi_{v_{!}}(j)}-\frac{\widetilde{l}^{j}}{\widetilde{\phi}_{v_{!}}(j \mid u)}\right) \log \left(1+\frac{u \mathrm{SNR}_{p}}{1+j \mathrm{SNR}_{c}}\right) \stackrel{(a)}{>} 0 \\
& \Rightarrow\left(\frac{1}{C}-\frac{1}{\widetilde{C}_{c}(u)}\right) \log \left(1+u \mathrm{SNR}_{p}\right)+\frac{1}{C \widetilde{C}_{c}(u)} \sum_{j=1}^{\infty}\left(\frac{\widetilde{C}_{c}(u) l^{j}}{\phi_{v_{!}}(j)}-\frac{C \widetilde{l}^{j}}{\widetilde{\phi}_{v_{!}}(j \mid u)}\right) \log \left(1+\frac{u \mathrm{SNR}_{p}}{1+j \mathrm{SNR}_{c}}\right) \stackrel{(b)}{>} 0
\end{aligned}
$$

In (a), we have made use of the fact that since $\widetilde{C}_{c}(u)>C>1$, multiplying a positive quantity by another one $\left(\widetilde{C}_{c}(u)\right)$ maintains the inequality. Likewise, in (b), multiplying a negative quantity by $C<\widetilde{C}_{c}(u)$ also keeps the sign of the inequality intact. By rearranging terms and regrouping according to cognitive or no cognitive case,

$$
\begin{aligned}
& {\left[\sum_{j=0}^{\infty} \frac{l^{j}}{C \phi_{v !}(j)} \log \left(1+\frac{u \mathrm{SNR}_{p}}{1+j \mathrm{SNR}_{c}}\right)\right]-\left[\sum_{j=0}^{\infty} \frac{\widetilde{l}^{j}}{\widetilde{C}_{c}(u) \widetilde{\phi}_{v !}(j \mid u)} \log \left(1+\frac{u \mathrm{SNR}_{p}}{1+j \mathrm{SNR}_{c}}\right)\right]>0} \\
& \Rightarrow \sum_{v=0}^{\infty}\left[p_{c}(v) \Psi(u, v)-\widetilde{p}_{v}(v \mid u) \Psi(u, v)\right]>0 \Rightarrow \phi_{p}(u)>\widetilde{\phi}_{p}(u)
\end{aligned}
$$




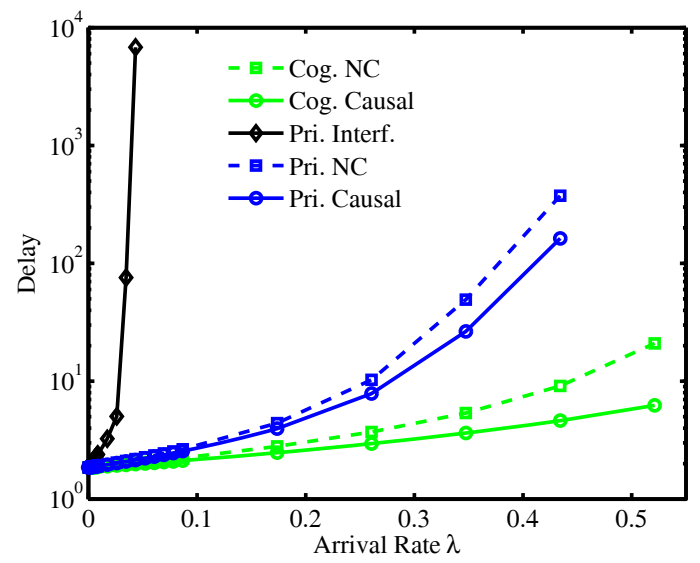

Fig. 2. Plot of Queuing Delay v/s the arrival rate for primary and cognitive user for causal, non-causal(NC) and mutual interference (no cognitive behavior) scenarios. SNR of both the users is $30 \mathrm{~dB}$.

of the queue [10]. Thus, larger loading factor(arrival rates) can be supported for both users with genie aided information than otherwise.

\section{NUMERICAL ANALYSiS}

We now evaluate the queuing delay for the primary and the cognitive user. For the non-causal case with the cognitive user having genie information of the primary user's codeword, we use (15) and (19) to evaluate the service rates for either user. We then make use of (9) to obtain the queuing delay. We consider the case when the arrival rates and consequently the loading factor $l$ are the same for both users. $\gamma_{p}=\gamma_{c}=1$.

In Fig.2, we plot the Delay as a function of the arrival rate $\lambda$ for a SNR of $30 \mathrm{~dB}$. Both the primary and the cognitive user have the same arrival rate and received SNR. In general, as the arrival rate increases, the queuing delay also increases as more packets queue up for service. From Fig.2, with cognitive behavior, both the users see a decrease in queuing delay. The cognitive behavior allows the primary and the cognitive users to support a larger arrival rate. With cognitive behavior and choice of parameters, $\lambda<0.6$ for the queue to be stable.

In Fig.3, the delay for the Gaussian cognitive radio is plotted for non-causal, causal and no cognition scenarios. The arrival rate is chosen as 0.0493 messages/second with a demand of $S=11$, corresponding to $P_{e}=10^{-3}$ and $K_{p}=K_{c}=100$. Both the primary and the cognitive users have the same power levels and arrival rates. We observe that the delay of the primary user has decreased with cognitive behavior. Significantly, as the SNR increases, the delay of the primary user is the same as that of the cognitive user with complete codeword information of the primary user.

In both Fig. 2 and Fig. 3, we have plotted the performance of a causal cognitive radio according to protocol 1 of [3]. In this protocol, the primary user allocates a portion of its power to send information to the cognitive transmitter during a broadcast phase while using the remaining power

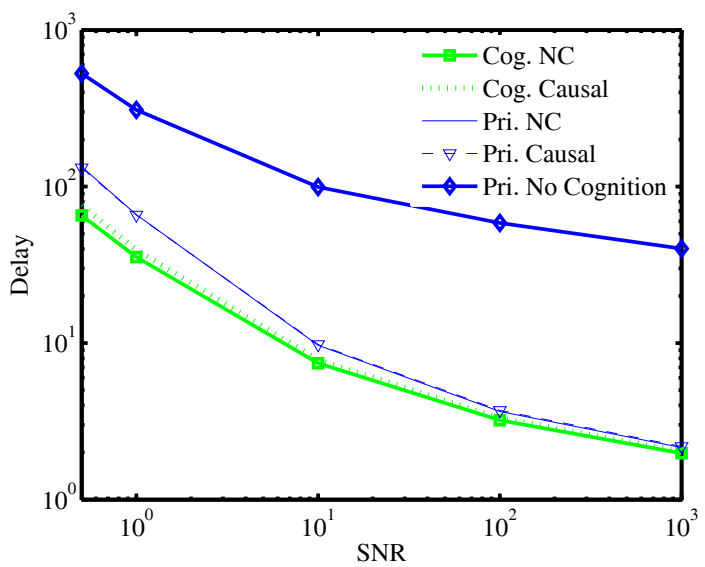

Fig. 3. Plot of Delay v/s SNR. The primary user's curve for causal and non-causal(NC) case are indistinguishable in this scale.

to transmit its information to its receiever. The cognitive user is silent during this broadcast phase. Following this, in the next transmission phase, the cognitive user does a dirty paper coding and achieves rates as if the primary user is absent while the primary user transmits with interference from the cognitive user. For paucity of space, we have omitted the analysis which is given in [5]. We observe the queuing delay for the causal scenario closely follows the non-causal case.

\section{CONCLUSION}

For a non-causal Gaussian cognitive radio with Poisson arrivals, we delineated a dirty paper like coding scheme that not only allows the cognitive user to transmit at rates as if the primary user is absent, but also improves the performance of the primary user. Similar performance is seen for a causal Gaussian cognitive radio. It would be interesting to verify the above behavior for other queuing models.

\section{REFERENCES}

[1] M. H. M. Costa, "Writing on dirty paper," IEEE Trans. Info. Theory, vol. 29, pp. 439-441, 1983.

[2] T. M. Cover and J. A. Thomas, Elements of Information Theory. Wiley, 1991.

[3] N. Devroye, P. Mitran, and V. Tarokh, "Achievable rates in cognitive radio channels," IEEE Tran. Info. Theory, vol. 52, pp. 1813-1827, 2006.

[4] A. Jovicic and P. Viswanath, "Cognitive radio: An information-theoretic perspective," submitted to IEEE Trans. Info. Theory, 2007.

[5] S. N. Kizhakkemadam and D. Rajan, "Share and gain: Random message arrivals in a gaussian cognitive radio," to be submitted to IEEE Trans. Info. Theory, 2007.

[6] R. Loynes, "The stability of a queue with non-independent inter-arrival and service times," Proc. Camb. Philos. Soc., vol. 58, pp. 497-520, 1962.

[7] J. Mitola, "Cognitive radio: An integrated agent architecture for software defined radio," Ph.D. dissertation, Royal Institute of Technology (KTH), Sweden, 2000.

[8] S. Srinivasa and S. A. Jafar, "The throughput potential of cognitive radio: A theoretical perspective," in Fortieth Asilomar Conference on Signals, Systems and Computers, Oct. - Nov 2006, pp. 221-225.

[9] I. E. Telatar, "Combining queueing theory with information theory for multi-access with joint decoding," in IEEE ITW, 1995, p. 51.

[10] I. Telatar and R. Gallager, "Combining queueing theory with information theory for multiaccess," Selected Areas in Communications, IEEE Journal on, vol. 13, no. 6, pp. 963-969, Aug. 1995. 